to all recruits on entry. Sulphonamides are not effective in the prevention of initial attacks.

As to the best method of treating an attack of rheumatism, there is still no unanimity of opinion. One of the most carefully undertaken and scrupulously recorded clinical trials was that undertaken by a joint Anglo-American team in 1951-2. The team issued a report after one year ${ }^{2}$ and a further one five years later. ${ }^{3}$ Now in the B.M.F. this week they give an account of their findings after 10 years (page 607).

Briefly, this trial was designed to compare the relative value of A.C.T.H., cortisone, and aspirin in the treatment of acute rheumatism. Of 497 children treated-240 from the United Kingdom and 257 from the U.S.A. - at the end of 10 years 420 had been traced, including 23 who were known to have died. 162 children received A.C.T.H., 167 cortisone, and 168 aspirin; the three groups were fairly well balanced as to age, sex, time of starting treatment, and severity of symptoms. In $51 \%$ treatment had been begun within two weeks of the onset of the attack and in nearly two-thirds there was no previous history or evidence of rheumatic fever. Strict diagnostic criteria were laid down and each treatment schedule lasted six weeks.

2. At the start of treatment 117 children had normal hearts ; 252 had carditis which was thought to have developed during the current attack; and 128 had carditis resulting from previous rheumatic heart disease. At the end of the first year there was no significant difference between the three treatment groups in the state of their hearts. Mortality was notably low. After five years there had been 14 deaths from rheumatic carditis (3\%). Of these children, 4 had first developed carditis during their initial period of treatment and the remaining 10 had already been affected from a previous attack. In $96 \%$ of the children whose hearts were normal at the beginning of treatment there was no residual carditis. In the 10-year follow-up it is striking that only five patients had died from carditis during the five years intervening since the last report five years ago. The low incidence of mitral stenosis is also noteworthy; there were only 18 cases among the 347 patients examined. The conclusion reached in the first two reports, that there was no evidence of superiority of any of the three treatment schedules, is confirmed in the 10-year report.

In a smaller though well-controlled trial undertaken by a combined study group in eight American paediatric centres in $1956^{4}$ a comparison was made between the results obtained by giving either prednisone $60 \mathrm{mg}$. daily for three weeks, followed by gradual reduction, or $50 \mathrm{mg}$. of aspirin per $\mathrm{lb}$. body weight per day for nine weeks to a series of children suffering from their first attack of unequivocal rheumatic carditis. The findings were that a large proportion of those with severe carditis recovered without residual damage but that others with similarly severe lesions did not. There was no significant difference between the two treatment schedules.

In contrast are the findings of May Wilson," who considers that prompt intensive short-term steroid treatment can influence the outcome in acute rheumatic carditis. She recommends a daily dose of cortisone $300-400 \mathrm{mg}$, or prednisone 100-200 mg., for about seven days (on the average), assessing the effective dose by the eosinophilic response and regarding more than 10 cells per c.mm. as

\footnotetext{
1 Stollerman, G. H., Pediat. Clin. N. Amer., 1964, 11, 213.

2 Brit. med. F., 1955, 1, 555.

3 Ibid., 1960, 2, 1033.

- Combined Rheumatic Fever Study Group, New Engl. f. Med., 1960, 262, 895 .

s Wilson, M. G., Advanc. Pediat., 1960, 11, 243.
}

indicative of inadequate therapy. Her observations, she concludes, show that in children with progressive clinical symptoms of active carditis this treatment will terminate the inflammatory process, significantly shorten its duration, and prevent or minimize residual cardiac damage. The important difference between her principles of therapy and those in the controlled trials by the Anglo-American team are that she gave much larger doses of steroids for a shorter period and varied the dose not according to the weight or to any specified levels (as required in a trial) but by the patient's response. Unfortunately her opinion is a minority one. If the work could be generally confirmed it would be a great advance, because it would represent a feature hitherto lacking in steroid therapy-namely, the ability to terminate rather than merely suppress inflammatory processes.

Most writers have agreed that there is no single regimen which can be accepted as of critical importance in influencing the outcome. Stollerman ${ }^{1}$ expresses the general view in saying that salicylates should be used when there are no signs of carditis, that steroids are usually given when there is mild carditis despite the doubt whether they are superior to salicylates, and that patients with severe carditis are given steroids promptly.

Finally, with regard to the prevention of recurrences, with their added risk of further cardiac damage, there is overwhelming evidence that penicillin, either by mouth as phenoxymethyl penicillin $125 \mathrm{mg}$. twice daily or intramuscularly as benzathine penicillin 1.5 million units monthly, or a sulphonamide such as sulphadimidine or sulphadiazine 0.5-1 g. daily by mouth, will be effective in the majority of patients for as long as it is continued. It is recommended that this prophylactic regimen should be followed for a minimum of five years. It should be stressed that this choice of prophylactics applies only to recurrences.

\section{Vascular Lesions of Diabetes Mellitus}

Since the two major hazards of diabetes mellitus, ketosis and intercurrent infection, can now be controlled effectively, it is the vascular complications of the disease that give doctors the most concern. They present the greatest danger to life, and cause many of the disabling symptoms.

The fundamental vascular lesion is called diabetic microangiopathy, as it affects the smallest arteries and the arterioles, venules, and capillaries. It consists of a proliferation of the endothelium of the intima and a thickening of the basement membrane, which by histochemical techniques is found to have a high polysaccharide content. This lesion is the basis of diabetic retinopathy, ${ }^{1}$ but the mode of formation of the typical microaneurysms seen with the ophthalmoscope is not understood. ${ }^{2}$ Diabetic glomerulosclerosis is another expression of the microangiopathy. ${ }^{3}$ It most often affects the glomerulus diffusely, in which case a uniform deposit of hyaline material, rich in polysaccharide, forms in and around the walls of the capillaries and progressively occludes their lumina. Less commonly, discrete hyaline nodules surrounded by capillaries appear in the glomerular tuft; this is the Kimmelstiel-Wilson lesion, ${ }^{4}$ which is diagnostic of diabetes mellitus. ${ }^{3}$ The diffuse lesion is associated with a large amount of protein in the urine, and by interfering with glomerular filtration it eventually leads to 
renal failure. The hypertension which is an integral part of diabetic nephropathy causes hyalinization of the media of the arterioles, especially the afferent glomerular ones. Microangiopathy affecting the nutrient vessels to the nerve fibres produces irreversible neuropathy. Similar lesions have been demonstrated in the skin, ${ }^{5}$ and are a possible explanation for the high incidence of superficial infection.

Diabetics are very prone to atheroma of the large arteries. This develops early and is more advanced in extent than in non-diabetic subjects of the same age. ${ }^{6}$ The cause of this premature atheroma is unknown. Though even wellcontrolled patients have slightly raised levels of non-esterified fatty acids and triglycerides in the plasma, ${ }^{7}$ the increased incidence of atheroma is unlikely to be due to a disturbance in lipid metabolism. ${ }^{8}$ Perhaps microangiopathy of the vasa vasorum of the larger arteries is an important factor.

The relation between diabetes mellitus and peripheral vascular disease is complex. In one series it was noted that diabetics did not come to operation at a significantly earlier age than did non-diabetic patients with arterial disease, ${ }^{9}$ and it was doubted whether peripheral vascular disease was influenced by diabetes. ${ }^{1 ;}$ Most workers think, however, that diabetics are much more prone to ischaemic lesions of the lower limbs than are non-diabetic subjects. ${ }^{6}$ Atheroma of the large blood-vessels, neuropathy causing the patient to neglect minor traumatic lesions, and a generalized lowering of resistance to infection are all important predisposing factors. ${ }^{11}$ Nevertheless, gangrene sometimes occurs in limbs free of severe infection and with palpable arterial pulses at the knee and even at the ankle-joint. In their recent paper in the B.M.F. Drs. J. M. Moore and I. D. O. Frew ${ }^{12}$ described the results of an examination of the blood-vessels of the skin of the lower limb in 52 diabetics and 52 non-diabetic controls. They found microangiopathy in 46 of the diabetics and in only 3 of the controls. Furthermore, of 34 diabetics with lesions of the foot 21 did not have clinically severe atheroma of the large arteries, whereas microangiopathy was absent in only 4 of them. This emphasizes the importance of occlusive microangiopathy in the aetiology of the foot lesions seen in diabetes mellitus. ${ }^{13}$

Microangiopathy begins early in the course of diabetes mellitus. It occurs in the initial latent phase, ${ }^{7}$ when abnormalities in the glucose-tolerance test are provoked only by external stress or by special laboratory procedures. ${ }^{14}$ Though it progresses in intensity - and may be virtually universal in long-standing diabetes mellitus-it gives rise to clinical effects in only a minority of patients. It has been noted in diabetes secondary to pancreatic disease, and is probably the result of

${ }^{1}$ Ashton, N., Brit. med. F., 1957, 1, 1002.

Lancet, 1959, 2, 625

${ }^{3}$ Gellman, D. D., Pirani, C. L., Soothill, J. F., Muehrcke, R. C., and Kark, R. M., Medicine (Baltimore), 1959, 38, 321.

- Kimmelstiel, P., and Wilson, C., Amer. F. Path., 1936, 12, 83.

5 Handelsman, M. B., Morrione, T. G., and Ghitman, B., Arch. intern. Med., 1962, 110, 70 .

6 Bell, E.'T Diabetes Mellitus, 1960. Thomas, Springfield, Ill

' Forsham, P. H., and Grodsky, G. M., in Biochemical Disorders in Human Disease, p. 657, 2nd ed. Ed. by R. H. S. Thompson and E. J. King,
orsham, P. H., and Grodsky, G. M., in Biochemical Disorders in Human 1964. Churchill, London.

${ }^{-}$Allen, E. V., Barker, N. W., and Hines, E. A., Peripheral Vascular Diseases, 3rd ed., 1962. Saunders, Philadelphia.

- Martin, P., in Peripheral Vascular Disorders, ed. by P. Martin, R. B. Lynn, J. H. Dible, and I. Aird, 1956. Livingstone, Edinburgh.

10 Dible, J. H., Lancet, 1958, 1, 1031.

${ }^{11}$ Oakley, W., Catterall, R. C. F., and Martin, M. M., Brit. med. F., 1956, 2, 953 .

1: Moore, J. M., and Frew, I. D. O., ibid., 1965, 2, 19.

1.3 Goldenberg, S., Alex, M., Joshi, R. A., and Blumenthal, H. T., Diabetes, 1959, 8, 261.

${ }_{14}^{14}$ EutzGerald, M. G., and Keen, H., Brit. med. F., 1964, 1, 1568.

"Gilliland, I. C., Hanno, M. G., and Strudwick, J. I., Biochem. F., 1954, 56, XXXII. an abnormality in metabolism due to an inadequate amount of insulin. But it may progress even in well-controlled patients. Raised levels of serum glycoproteins have been noted in diabetics with vascular lesions, ${ }^{1516}$ and possibly microangiopathy is due to a seepage of polysaccharides from the circulation through foci of capillary damage. ${ }^{1}$ Nevertheless we still have much to learn about a very important aspect of diabetes mellitus.

\section{Treatment of Lung Cancer}

Resection is still the best treatment for bronchial carcinoma. Radiotherapy, although of great value for the relief of symptoms, cannot match the five-year cure rate of the surgeons, ${ }^{1}$ nor does it seem to have much to offer when used in combination with surgery ; indeed, it may have risks of its own. Chemotherapy with cytotoxic drugs such as nitrogen mustard, vinblastine, ${ }^{2}$ or other newer preparations is of little value whether they have been given on their own or in conjunction with surgery.

There is now fairly wide agreement that conservative resection in the form of lobectomy, where it is possible, offers as good a chance as the more radical pneumonectomy. The proportion of patients in whom it is done is probably more than a third of the total, and it has the advantage that the operative mortality is less than half that of pneumonectomy.

The incidence of bronchial carcinoma in Britain has risen inexorably year after year for over a decade, and 25,000 deaths were attributed to it in England and Wales in $1963{ }^{3}$ It is thus by far the commonest carcinoma affecting men, and accounts for $40 \%$ of the deaths of males from malignant disease. Even so on average any one general practitioner is likely to see only one patient with bronchial carcinoma each year. This emphasizes the great vigilance required to diagnose the condition early and thus to refer patients for treatment at a stage where there is some prospect of success.

It is difficult to find out what proportion of patients suffering from bronchial carcinoma are suitable for surgery, but probably only one-fifth of them fall into this group; in about three-quarters of these resection proves possible: The length of history of those who have been operated on does not appear to affect the results. ${ }^{4}$ However, if more patients have their disease recognized early enough to be found suitable for surgery, then a larger number will benefit from it.

Recent papers have shown that the five-year survival rate after resection for bronchial carcinomas is about $25 \%,,^{5-7}$ and in some series it has been notably higher than this. ${ }^{8}$ The figure compares favourably with the results of the surgical treatment of another common carcinoma-that of the stomach -in which few surgeons claim a five-year survival rate of more than $10 \%$. On the other hand the survival rates after surgery for carcinoma of the breast or the rectum are considerably better-in the region of $40 \%$. Despite a recent fall

Morrison, R., Deeley, T. J., and Cleland, W. P., Lancet, 1963, 1, 683. Crosbie, W. A., Kamdar, H., and Belcher, J. R., Brit. F. Dis. Chest, 1965, in press.

${ }^{3}$ The Registrar-General's Statistical Review of England and Wales for the Year 1963, Part I. H.M.S.O. 1965.

Rienhoff, W. F., Talbert, J. L., and Wood, S., Ann. Surg., 1965, 161, 674.

Belcher, J. R., and Anderson, R., Brit. med. F., 1965, 1, 948.

- Goldman, K.'P., Thorax, 1965, 20, 298.

Clagett, O. T., Allen, T. H., Payne.W. S., and Woolner, L. B., f. thorac. cardiovasc. Surg., 1964, 48, 391.

Flavell, G., Brit. med. f., 1962, 1, 284.

Capel, L. H., unpublished data. 